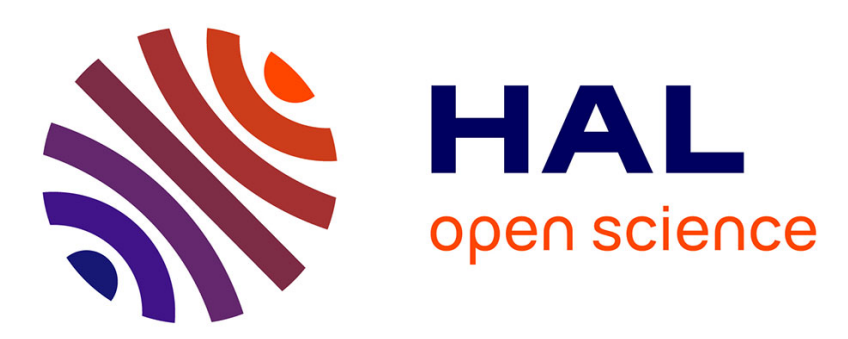

\title{
Approximation Methods to Solve Stochastic Problems in Computational Electromagnetics \\ Stéphane Clénet
}

\section{To cite this version:}

Stéphane Clénet. Approximation Methods to Solve Stochastic Problems in Computational Electromagnetics. Mathematics in Industry - Scientific Computing in Electrical Engineering, 23, Springer International Publishing, pp.199-214, 2016, 978-3-319-30399-4. 10.1007/978-3-319-30399-4_20 . hal03165202

\section{HAL Id: hal-03165202 \\ https://hal.science/hal-03165202}

Submitted on 10 Mar 2021

HAL is a multi-disciplinary open access archive for the deposit and dissemination of scientific research documents, whether they are published or not. The documents may come from teaching and research institutions in France or abroad, or from public or private research centers.
L'archive ouverte pluridisciplinaire HAL, est destinée au dépôt et à la diffusion de documents scientifiques de niveau recherche, publiés ou non, émanant des établissements d'enseignement et de recherche français ou étrangers, des laboratoires publics ou privés. 


\title{
Approximation Methods to solve Stochastic Problems in Computational Electromagnetics
}

\author{
Stéphane Clénet
}

\begin{abstract}
To account for uncertainties on model parameters, the stochastic approach can be used. The model parameters as well as the outputs are then random fields or variables. Several methods are available in the literature to solve stochastic models like sampling methods, perturbation methods or approximation methods. In this paper, we propose an overview on the solution of stochastic problems in computational electromagnetics using approximation methods. Some applications will be presented in order to illustrate the possibilities offered by the approximation methods but also their current limitations due to the curse of dimensionality. Finally, recent numerical techniques proposed in the literature to face the curse of dimensionality are presented for non-intrusive and intrusive approaches.
\end{abstract}

\section{Introduction}

Applying a discretisation scheme (Finite Element Method-FEM, Finite Integration Technique-FIT, ...) to solve the Maxwell equations leads to valuable tools for understanding and predicting the features of electromagnetic devices. With the progress in the fields of numerical analysis, CAD and postprocessor tools, it is now possible to represent and to mesh very complex geometries and also to take into account more realistic material behaviour laws with non-linearities, hysteresis .... Besides, computers have nowadays such capabilities that it is common to solve problems with millions of unknowns. The modelling error due to the assumptions made to build the mathematical model (the set of equations) and the numerical errors due especially to the discretisation (by a FEM for example) can be negligible. Consequently, in some applications represented by very accurate models (the modelling and the numerical errors are negligible), if a gap exists between the measurements, assum-

Stéphane Clénet

L2EP, Arts et Métiers ParisTech, 8, Bd Louis XIV, 59046 Lille cedex, France, e-mail: stephane.clenet@ensam.eu 
ing perfect, and the results given by the numerical model, it comes from deviations on input parameters which are not in the "real world" equal to their prescribed values. The origins of these deviations are numerous and are related to either a lack of knowledge (epistemic uncertainties) or uncontrolled variations (aleatoric uncertainties). For example, mechanical parts are manufactured with dimensional tolerances whereas some dimensions, such as air gaps in electric machines, are critical as they strongly influence performance. Besides uncertainties in material composition, the material characteristics which change with uncontrolled environmental factors (humidity, pressure, etc.) are also often unknown [39]. Even if the environmental factors are perfectly known, in some situations, the behaviour law parameters cannot be identified because measurements are not possible under the right experimental conditions. Consequently, to be more realistic, numerical models must now be able to take into account uncertainties.

The stochastic approach which consists in representing the uncertain parameters as random variables, (the output variables are then also random variables) is one possible way to model and to evaluate the influence of the uncertainties on the parameters. Monte Carlo Simulation methods or perturbation methods are available to solve stochastic problems since early $50^{\prime} \mathrm{s}$ [11,12]. In the $90^{\prime}$ s, researches on quantification of uncertainties in numerical models using approximation methods first began in the field of mechanical and civil engineering [31]. In the 2000's, this approach has met a growing interest with the development of approximation methods based especially on truncated polynomial chaos expansions that offer a higher convergence rate than the Monte Carlo Simulation Method if the model outputs present a sufficient regularity versus the input parameters.

In this paper, we propose a survey on the solution of stochastic problems in computational electromagnetics using approximation methods. First, we present the deterministic model based on FEM then the stochastic model is derived when the input parameters are considered as random variables. The approximation method is introduced which consist in finding a solution in a finite dimensional functional space. Different numerical techniques, available in the literature, are described to solve the stochastic problem. Then, a description of applications of the stochastic approach in the field of computational electromagnetics is proposed in order to illustrate the capabilities of such approach but also its current limitations particularly due to the curse of dimensionality. Finally, recent numerical techniques proposed in the literature to face the curse of dimensionality are presented.

\section{Presentation of the Problem}

\subsection{Deterministic Problem}

In the following, we will address the magnetostatic problem but the results can be easily extended to other static and quasi static field problems. In the following, the 
aim is to introduce notations when the magnetostatic problem is solved numerically using the vector potential formulation and FEM. The partial differential equations to be solved on a domain $\mathrm{D}$ are:

$$
\begin{gathered}
\operatorname{curl} \mathbf{H}(\mathbf{x})=\mathbf{J}(\mathbf{x}) \\
\operatorname{div} \mathbf{B}(\mathbf{x})=0
\end{gathered}
$$

with $\mathbf{H}$ the magnetic field, $\mathbf{B}$ the magnetic flux density and $\mathbf{J}$ the current density that is assumed to be known. In addition, boundary conditions on $\mathbf{H}$ and $\mathbf{B}$ are added and also the behaviour law of the material which will be assumed to be written in the form:

$$
\mathbf{H}(\mathbf{x})=v(\mathbf{x}) \mathbf{B}(\mathbf{x})
$$

with $v$ the reluctivity. To solve the problem, the vector potential formulation can be used:

$$
\operatorname{curl}[v(\mathbf{x}) \operatorname{curl} \mathbf{A}(\mathbf{x})]=\mathbf{J}(\mathbf{x})
$$

with $\mathbf{A}$ the vector potential. To find an approximate solution of this equation, FEM is often applied. We seek for an approximation $\mathbf{A}$ of the vector potential in the edge element space such that:

$$
\mathbf{A}(\mathbf{x})=\sum_{i=0}^{\mathrm{N}} a_{i} \mathbf{w}_{i}(\mathbf{x})
$$

with $\mathrm{N}$ the number of Degrees of Freedom (DoFs), $\mathbf{w}_{i}$ the edge shape functions and $a_{i}$ unknown real coefficients. By applying the Galerkin method to a weak form of (4):

$$
\int_{D} v(\mathbf{x}) \operatorname{curl} \mathbf{A}(\mathbf{x}) \cdot \operatorname{curl}_{i}(\mathbf{x}) d x=\int_{D} \mathbf{J}(\mathbf{x}) \mathbf{w}_{i}(\mathbf{x}) d x \quad \forall i \in[1 ; \mathbf{N}]
$$

Replacing $\mathbf{A}$ by its expression (5) in (6), a system of $\mathrm{N}$ linear equations with $\mathrm{N}$ unknown coefficients $a_{i}$ is obtained which can be written in the form:

$$
\mathbf{S} \mathbf{A}=\mathbf{F}
$$

with $\mathbf{S}$ the stiffness matrix $(\mathrm{NxN}), \mathbf{F}$ the source vector $(\mathrm{Nx} 1)$ and $\mathbf{A}$ the vector of the coefficients $a_{i}$. We should mention that non-homogeneous boundary conditions can be taken into account, additional entries are then added to the source vector $\mathbf{F}$. The coefficients $s_{i j}$ of $\mathbf{S}$ and $f_{i}$ of $\mathbf{F}$ satisfy:

$$
s_{i j}=\int_{D} v(\mathbf{x}) \operatorname{curlw}_{j}(\mathbf{x}) \cdot \operatorname{curlw}_{i}(\mathbf{x}) d x \quad f_{i}=\int_{D} \mathbf{J}(\mathbf{x}) \cdot \mathbf{w}_{i}(\mathbf{x}) d x
$$

Once the equation system (6) is solved, local quantities like the magnetic flux density distribution or global quantities of interest like the flux, the torque can be calculated in a post processing step. 


\subsection{Stochastic Problem}

In the deterministic case, the input parameters like the dimensions related to the geometry of the device, the material characteristics and the electromagnetic sources are supposed to be perfectly known. If the input parameters of the model are subject to variability, the solution of (7) will be also subject to variability. The stochastic approach enables to quantify this variability. When accounting for the uncertainties using the stochastic approach, the input parameters are then modelled by random variables $\mathbf{p}(\theta)$ with $\theta$ an elementary event. The joint probability density function is supposed to be known (or each marginal probability density functions if the random variables are independent). The outputs of the electromagnetic model become then random and should be characterized. A stochastic partial differential equation system is generally numerically solved by applying, like in the deterministic case (see 2.1), a semi-discretisation in space (see 6). The DoF's $a_{i}$ of the vector potential (see (5)), which were real numbers in the deterministic case, becomes random variables $a_{i}[\mathbf{p}(\theta)]$. The matrix $\mathbf{S}$ and the vector $\mathbf{F}$ have random entries $s_{i j}[\mathbf{p}(\boldsymbol{\theta})]$ and $f_{i}[\mathbf{p}(\boldsymbol{\theta})]$ and the unknown vector $\mathbf{A}$ is random and satisfies:

$$
\mathbf{S}[\mathbf{p}(\theta)] \mathbf{A}[\mathbf{p}(\theta)]=\mathbf{F}[\mathbf{p}(\theta)]
$$

As already mentioned above, the input parameters $\mathbf{p}(\theta)$ of the model are related either to the geometry or to the behaviour laws of the material or to the sources (including non-homogeneous boundary conditions). Taking into account the randomness on the source is quite straightforward especially when the deterministic problem is linear [4]. In the following, we will assume that the sources are deterministic. For the other kinds of randomness, the problem is more complicated. The processing of uncertain geometries is slightly different than the processing of uncertain behaviour laws and requires additional treatments. The most natural way to account for randomness on the geometry consist in remeshing according to the deformation but the remeshing leads to a discontinuous solution in the space of the input parameters and can create additional numerical noise which can disturb the random solution. Alternatives have been proposed in the literature [5-9] to avoid remeshing. In the following, we will focus mainly on uncertainties on the behaviour laws. However, the quantification methods presented in the following can be applied to solve problems with random geometries as mentioned previously.

To solve (9), sampling techniques, like the Monte Carlo Simulation Methods (MCSM) [12] [11], or perturbation methods [44] [45] can be applied. In this paper, we will focus only on the approximation methods which are well fitted to solve (9) when the entries of the vector $\mathbf{A}(\mathbf{p})$ are smooth functions of the input parameters $\mathbf{p}$. 


\section{Approximation methods}

We denote $G$ the quantity of interest which can be an unknown of the problem (a value of the circulation $a_{i}[\mathbf{p}(\theta)]$ of the vector potential along an edge $i$ ), a local quantity like the value of the magnetic field or the Joule losses at one point of the domain or a global quantity derived from the magnetic fields like the magnetic flux flowing through a stranded inductor or the magnetic energy. An approximation of the quantity $\mathrm{G}$ which is a function of the random input parameters $\mathbf{p}(\theta)$, is sought in a finite dimensional function space of $\mathbf{p}(\theta)$ that is to say:

$$
G[\mathbf{p}(\theta)]=\sum_{i=0}^{\mathrm{P}} g_{i} \Psi_{i}[\mathbf{p}(\theta)]
$$

with $g_{i}$ coefficients to determine. The approximation functions $\Psi_{i}[\mathbf{p}(\theta)]$ can be chosen in different finite dimensional spaces $[4,15]$. If the output $\mathrm{G}$ has a finite variance and is sufficiently smooth, polynomial expansions are well suited. If it exists some singularities (for example in the case random geometry), other approximation spaces should be introduced [13]. Approximations based on the Polynomial Chaos Expansion (PCE) are currently the most used in engineering. PCE was first introduced by Wiener [16] to represent Gaussian processes. In [17], Xiu et al. proposed a more general approach by referring to the Wiener-Askey scheme. A PCE requires the random components $p_{i}(\theta)$ of the vector $\mathbf{p}(\theta)$ to be independent. If it is not the case, alternatives are proposed in the literature either to modify the approximation space or to express the vector $\mathbf{p}(\theta)$ as a function of a vector $\mathbf{p}(\theta)$ of independent random variables (using isoprobabilistic transformation for example). In the following, we will assume the random variables $p_{i}(\theta)$ independent with a probability density function (pdf) $f_{i}(y)$. The size of the random vector $\mathbf{p}(\theta)$ will be equal to K. We denote $\mathrm{E}[\mathrm{X}(\theta)]$ the expectation of the random variable $\mathrm{X}(\theta)$ (the expectation of $X(\theta)$ is equal to the mean of $X(\theta)$ ). We introduce now the monovariate orthogonal polynomial $\psi_{i}^{l}(y)$ of order 1 associated to the parameter $p_{i}(\theta)$. The polynomials $\psi_{i}^{l}(y)$ are orthogonal with respect to the pdf $f_{i}(y)$ that is to say:

$$
E\left[\psi_{i}^{l}\left(p_{i}(\theta)\right) \psi_{i}^{m}\left(p_{i}(\theta)\right)\right]=\int_{-\infty}^{+\infty} \psi_{i}^{l}(y) \psi_{i}^{m}(y) f_{i}(y) d y=\delta_{l m}
$$

with $\delta_{l m}$ the kronecker symbol. The determination of the monovariate polynomials $\psi_{i}^{l}(y)$ is not an issue whatever the pdf of $f_{i}(y)$ (see [17]). We define now the set of multivariate orthogonal polynomials $\Psi_{\alpha}[\mathbf{p}(\theta)]$ with $\alpha$ a K-tuple such that:

$$
\Psi_{\alpha}(\mathbf{p}(\theta))=\prod_{i=1}^{K} \psi_{i}^{\alpha_{i}}\left(p_{i}(\theta)\right) \text { with } \alpha=\left(\alpha_{1}, \ldots, \alpha_{K}\right) \alpha_{i} \in \mathrm{N}
$$

Since $\mathbf{p}(\theta)$ is a vector of independent random variables, the multivariate polynomials $\Psi_{\alpha}(\mathbf{p}(\theta))$ are orthogonal with respect to the joint probability density function $\prod_{i=1}^{K} f_{i}$ and we have $\mathrm{E}\left[\Psi_{\alpha}(\mathbf{p}(\theta)) \Psi_{\beta}(\mathbf{p}(\theta))\right]=0$ if $\alpha \neq \beta$. If the random variable 
$G[\mathbf{p}(\theta)]$ has a finite variance, the PCE refers to the representation of $G[\mathbf{p}(\theta)]$ as a linear combination of multivariate polynomials $\Psi_{\alpha}(\mathbf{p}(\theta))$ :

$$
G[\mathbf{p}(\theta)]=\sum_{\alpha_{1}=0}^{+\infty} \ldots \sum_{\alpha_{K}=0}^{+\infty} g_{\alpha} \Psi_{\alpha}[\mathbf{p}(\theta)]
$$

In practice, the expansion (13) is truncated up to the multivariate polynomials of order $\mathrm{p}$ (the sum $\alpha_{1}+\ldots+\alpha_{K}$ is lower or equal than $\mathrm{p}$ ). The total number of multivariate polynomials $\mathrm{P}$ to be considered is:

$$
\mathrm{P}=\frac{(\mathrm{K}+\mathrm{p}) !}{\mathrm{K} ! \mathrm{p} !}
$$

In Table 1, we have reported the number of multivariate polynomials $\mathrm{P}$ of the space of approximation as a function of the maximum polynomial order $\mathrm{p}$ and the number $\mathrm{K}$ of input parameters. We can see that $\mathrm{P}$ increases exponentially with $\mathrm{K}$ which is usually so-called the curse of dimensionality.

Table 1 Example of the multivariate polynomial number as a function of the maximum multivariate polynomial order $\mathrm{p}$ and the number of random inputs $\mathrm{K}$

\begin{tabular}{lllll}
\hline & $\mathrm{p}=1$ & $\mathrm{p}=2$ & $\mathrm{p}=3$ & $\mathrm{p}=4$ \\
\hline $\mathrm{K}=2$ & 3 & 6 & 10 & 15 \\
$\mathrm{~K}=5$ & 6 & 21 & 56 & 126 \\
$\mathrm{~K}=10$ & 11 & 66 & 286 & 1001 \\
$\mathrm{~K}=20$ & 21 & 231 & 1771 & 10626 \\
\hline
\end{tabular}

In the following, to simplify the notation, the multivariate polynomials $\Psi_{\alpha}(\mathbf{p}(\theta))$ will be indexed by an integer $i(1 \leq i \leq P)$ instead of the K-tuple $\alpha$. The function $G[\mathbf{p}(\theta)]$ is approximated by a truncated expansion given by (10) of orthogonal multivariate polynomials defined by (12). As already mentioned previously, after applying the semi-discretisation in space, the terms $a_{i}[\mathbf{p}(\theta)]$ of the decomposition of the vector potential $\mathbf{A}[\mathbf{x}, \mathbf{p}(\theta)]$ are random (see (5)). Each term $a_{i}[\mathbf{p}(\theta)]$ is approximated using a truncated PCE (13). Finally, the vector potential $\mathbf{A}[\mathbf{x}, \mathbf{p}(\theta)]$ is approximated by the expression:

$$
\mathbf{A}[\mathbf{x}, \mathbf{p}(\theta)]=\sum_{i=0}^{\mathrm{N}} \sum_{j=0}^{\mathrm{K}} a_{i j} \Psi_{j}(\mathbf{p}(\theta)) \mathbf{w}_{i}(\mathbf{x})
$$

The number of coefficients $a_{i j}$ is equal to NxP. It is not seldom to meet in practise deterministic models with a number of unknowns $\mathrm{N}$ of order $10^{5}$. According to (14) and Table 1 , the unknown number NxP can be quickly very huge (of order $10^{8}$ ) if the number $\mathrm{K}$ of random input parameters is higher than a dozen.

In a postprocessing step, quantities of interest (energy, flux,) can be also expressed using (10). Among the method proposed in the literature to determine these 
coefficients, some are called non-intrusive since they encapsulate a deterministic model in an environment of stochastic procedures. A preprocessor generates a sample of parameter values according to their probability density function. A deterministic model is then run for each set of parameter values of the sample and a new sample of output values is then obtained. From this sample, a postprocessor determines the approximation of the output. Collocation [32], regression [24] and projection methods belongs to this group of non-intrusive methods. Some stochastic methods, so-called intrusive methods, require to access to the heart of the deterministic model to be implemented like the Spectral Stochastic Finite Element Method. In the following, to illustrate the main principles of intrusive and non-intrusive methods, we will present the projection method and the Spectral Stochastic Finite Element Method.

\subsection{A non-intrusive method : Projection Method}

Since the polynomials $\Psi_{i}(\mathbf{p}(\theta))$ are orthogonal, the coefficients $a_{i j}$ satisfy:

$$
\begin{aligned}
a_{i j} & =\frac{\left.E\left[a_{i}(\mathbf{p}(\theta)) \Psi_{j}(\mathbf{p}(\theta))\right]\right]}{\left.E\left[\Psi_{j}^{2}(\mathbf{p}(\theta))\right]\right]} \\
& =\frac{\int_{-\infty}^{+\infty} \ldots \int_{-\infty}^{+\infty} a_{i}\left(p_{1}, \ldots, p_{K}\right) \Psi_{j}\left(p_{1}, \ldots, p_{K}\right) f_{1}\left(p_{1}\right) \ldots f_{K}\left(p_{K}\right) d p_{1} \ldots d p_{K}}{\int_{-\infty}^{+\infty} \ldots \int_{-\infty}^{+\infty} \Psi_{j}^{2}\left(p_{1}, \ldots, p_{K}\right) f_{1}\left(p_{1}\right) \ldots f_{K}\left(p_{K}\right) d p_{1} \ldots d p_{K}}
\end{aligned}
$$

The determination of $a_{i j}$ yields the calculation of multidimensional integrals. The denominator of (16) can be calculated generally analytically $\left(d_{j}=E\left[\Psi_{j}^{2}(\mathbf{p}(\theta))\right]\right)$ but not the numerator. Different methods can be used to approximate the multidimensional integral: MCSM, Gauss quadrature methods, sparse grid methods, adaptive integration schemes... [2] [20]. All of them yield the following expression for the approximation:

$$
a_{i j}=\frac{\sum_{l=1}^{Q} a_{i}\left(\mathbf{p}^{l}\right) \Psi_{j}\left(\mathbf{p}^{l}\right) w^{l}}{d_{j}}
$$

where $w^{l}$ are the weights and $\mathbf{p}^{l}=\left(p_{1}^{l}, \ldots, p_{K}^{l}\right)$ the $\mathrm{Q}$ evaluation points. The model (9) is solved for $\mathrm{Q}$ sets of the input parameters $\mathbf{p}^{l}$ to determine $a_{i}\left(\mathbf{p}^{l}\right)$ that is to say that the deterministic model (7) has to be solved Q times with $\mathbf{p}^{l}$ as input parameters. One should notice that Q can increase dramatically with $\mathrm{K}$. Let consider for example a Gauss quadrature of order $q_{i}$ along the random direction $i$ associated to each parameter $p_{i}(1 \leq i \leq K)$. We denote by $p_{i}^{l} 1 \leq l \leq q_{i}$ the evaluation points and $w_{i}^{l} 1 \leq l \leq q_{i}$ the associated weights. The points are the roots of the polynomial $\psi_{i}^{q}(y)$ of order q introduced in (11). A multidimensional quadrature can be obtained by tensorizing the monodimensional gauss quadratures along each random dimension. In that case, the number of evaluation points $\mathrm{Q}$ is equal to $q_{1} q_{2} \ldots q_{K}$ and so increases exponentially with the number $\mathrm{K}$ of parameters. The number of evalua- 
tion points can be reduced by using sparse grids like Smolyak cubature [23] but the exponential increasing with the dimension remains.

The coefficients $g_{i}$ of the approximation of any quantity of interest $G[\mathbf{p}(\theta)]$, like the flux or the force, can be determined using the same approach. If $\mathrm{G}$ is the only quantity of interest for the user, there is no need to access to the $a_{i}[\mathbf{p}(\theta)]$ 's. The deterministic model is run $\mathrm{Q}$ times, as a black box, with the different parameter values $\mathbf{p}^{l}$ to calculate $\mathrm{Q}$ evaluations $\mathrm{G}\left[\mathbf{p}^{l}\right]$. From the $\mathrm{G}\left[\mathbf{p}^{l}\right]$ 's, the coefficients $g_{i}$ are approximated using a quadrature formula (see 17). The non-intrusive approach is very convenient because the coupling with existing deterministic models, especially commercial software, is straightforward.

One should note that the non-linearities on the behaviour laws are naturally taken into account within the deterministic model that is to say that the non-intrusive method is the same when dealing with either a linear model or a non-linear model.

\subsection{Galerkin method : Stochastic Finite Element Method}

To solve stochastic partial differential equations, the Galerkin approach was first introduced in the early 90 s by Ghanem et al in mechanics [31]. It consists in searching the solution in a tensorial space $W(D) \otimes \mathrm{P}_{P}^{K}$ with $W(D)$ the standard finite element space used in the deterministic case and $\mathrm{P}_{P}^{K}$ the space of approximation of random variables spanned by the basis functions $\left(\Psi_{i}[\mathbf{p}(\theta)]\right)_{1 \leq i \leq P}$ introduced previously (see (10)). In magnetostatics, the vector potential is sought in a space generated by the basis function $\Psi_{j}(\mathbf{p}(\theta)) \mathbf{w}_{i}(\mathbf{x})$. The solution should satisfy a weak form of the initial problem. Let consider again our magnetostatic problem, the weak form (6) is extended in the stochastic case and can be written [26]:

$$
\begin{array}{r}
E\left[\int_{D} v(\mathbf{x}, \mathbf{p}(\theta)) \operatorname{curl} \mathbf{A}(\mathbf{x}, \mathbf{p}(\theta)) \cdot \mathbf{c u r l w}_{i}(\mathbf{x}) d x \Psi_{j}(\mathbf{p}(\boldsymbol{\theta}))\right] \\
=E\left[\int_{D} \mathbf{J}(\mathbf{x}) \mathbf{w}_{i}(\mathbf{x}) d x \Psi_{j}(\mathbf{p}(\theta))\right] \quad \forall i \in[1 ; \mathrm{N}] \quad \text { and } \quad \forall j \in[1 ; \mathrm{P}]
\end{array}
$$

Replacing $\mathbf{A}[(x), \mathbf{p}(\theta)]$ in (18) by its expression (15) and applying the weak formulation for the NxP test functions $\Psi_{j}(\mathbf{p}(\theta)) \mathbf{w}_{i}(\mathbf{x})$, a NxP equation system is obtained:

$$
\mathbf{S}_{s} \mathbf{A}_{s}=\mathbf{F}_{s}
$$

with $\mathbf{S}_{s}$ a $(\mathrm{NxP}) \mathrm{x}(\mathrm{NxP})$ matrix, $\mathbf{A}_{s}$ the (NxP) vector of the unknowns $a_{i j}$ and $\mathbf{F}_{s}$ a (NxP) vector. The "intrusivity" of the method is related to the fact that the entries of $\mathbf{S}_{s}$ and $\mathbf{F}_{s}$ are integral functions of $\Psi_{j}(\mathbf{p}(\theta))$ and $\mathbf{w}_{i}(\mathbf{x})$. Their calculation requires to have access to the procedures of the calculation of the terms $s_{i j}$ and $f_{i}$ (see (8)) of the deterministic model. The size of the system (NxP) can be extremely large preventing the storage of the matrix $\mathbf{A}_{s}$ and so its solution. If the reluctivity can be written as a sum of separable functions like, 


$$
v(\mathbf{x}, \mathbf{p}(\theta))=\sum_{i=1}^{M} v_{i}[\mathbf{p}(\theta)] g_{i}(\mathbf{x})
$$

the system (19) can be rewritten taking advantage of the Kronecker product [46]. This representation of the reluctivity as a sum of separable functions can be obtained either during the process of probabilistic modelling of the input data or by applying a model reduction technique (Karuhnen-Loeve expansion for example). According to this new expression, the matrix $\mathbf{S}_{s}$ can be written in the form [3]:

$$
\mathbf{S}_{s}=\sum_{i=1}^{M} \mathbf{C}_{i} \otimes \mathbf{D}_{i}
$$

The memory space required can be significantly reduces by storing only the $2 \mathrm{M}$ matrices $\mathbf{C}_{i}$ and $\mathbf{D}_{i}$ with $\mathbf{C}_{i}$ depending only on the functions $\mathbf{w}_{i}(\mathbf{x})$ and $\mathbf{D}_{i}$ on the functions $\Psi_{j}(\mathbf{p}(\theta))$. It should be noticed that the matrices $\mathbf{C}_{i}$ can be easily extracted from a deterministic standard finite element code. The determination of the matrix $\mathbf{S}_{s}$ does not require a high modification of the deterministic code and so the intrusivity of the Galerkin approach can be highly alleviated using expression based on separable functions. This approach can be extended to quasistatics. Besides, dedicated solvers can be employed to solve the equation (19) by taking advantage the expression (21) based on Kronecker products. Accounting for non-linearities in the Galerkin approach is more tricky than in the non-intrusive case but remains possible [28]. The Galerkin method, for given approximation spaces $W(D)$ and $\mathrm{P}_{P}^{K}$, minimizes the error of approximation in the L2 sense which is not the case with other approximation methods based on the evaluations of the deterministic model (non intrusive methods like projection method, collocation method, regression method). However, when a multivariate double orthogonal polynomial expansion is used to approximate the stochastic dimension then the collocation and the Galerkin methods are equivalent [26].

\section{Applications}

Approximation methods have been already applied in computational electromagnetics to study EEG Source Analysis [19], Eddy Current in human body [18], Eddy Current Non Destructive Testing [1] [2], Accelerator Cavities and Magnets [21] [47] [48], Dosimetry [22], electrical machines [29] [30] The development and the application of such models have started in the early 2000 's and know a growing interest in the community. The methods have been evaluated on academic examples [26,28] but one can notice a trend towards more and more realistic applications which shows that the stochastic approach is getting more and more mature in the community of computational electromagnetics. In [19], the projection method has been applied uncertainties in the EEG source analysis. In [32], a 2D dosimetry problem has been tested by comparing several non-intrusive approximation methods and the Monte 
Carlo Simulation Method. It has been shown that the approximation methods enable to reduce dramatically the number of evaluation points compared to sampling technics. An Eddy Current-Non Destructive Testing problem where some material characteristics are assumed to be random has been addressed. Samples were not accessible for measurement (nuclear application) [34] to determine the conductivity and the permeability of material like magnetite deposit. The lack of knowledge was modelled by a stochastic approach considering the material characteristic as random variables. The aim was to determine the influence of this lack of knowledge on the model output, here the output sensor. In this application, a sensitivity analysis showed that only one material characteristic among the 6 considered has an influence on the variability of the sensor output. In other words, only the lack of knowledge of one material characteristic ( $p$ ) has an influence on the accuracy of the model. Consequently, to improve the accuracy, investigation shall focus on the parameter $p$ and not on the others. This study shows that the stochastic approach is a powerful tool for improving the accuracy of models by determining the input parameters whose uncertainties (due to a lack of knowledge) strongly influence the quantity of interest. It can also be very helpful to develop indicators based on measurements that are robust, that is to say that these indicators are few influenced by the variability introduced by the imperfections on the device studied. To solve this problem, the Galerkin method and an Projection method are compared [1] [2]. It shows that the Galerkin approach can be competitive compared to a non-intrusive approach. The influence of the lack of knowledge on the $\mathrm{B}(\mathrm{H})$ curve of the ferromagnetic material has been also addressed in the case of a turbo alternator [35]. The global sensitivity analysis based on the Sobol approach [50-52] allows to determine the most influential parameters of the $\mathrm{B}(\mathrm{H})$ curve. It appears that the magnetic flux density is the most influential but not the magnetic field $\mathrm{H}$ in the saturation area. The proposed approach provides the quantity of interest domain where the parameter uncertainties are the most influential and then allows to act in order to reduce their variability by increasing the accuracy of the measurement in the corresponding area.

The influence of the dimension and material characteristics variability on the performances of an electrical machines produced in mass is also studied when the number of random parameters is about a dozen $[29,30]$. The aim is to propose a methodology based on a stochastic approach to assess the influence of the variability of the manufacturing process on the performances of the electrical machines which can be applied in robust design. The tolerancing using the stochastic approach has been also studied for a permanent magnet machine [36].

\section{Facing the curse of dimensionality}

If we want to go further with the stochastic approach which can be very useful to solve numerous problems in engineering, the curse of dimensionality should be overcome in order to be able to deal with real world problems where the number of 
parameters is often greater than the dozen. In the following, we will present briefly methods that have been proposed recently to overcome this challenge. We will keep the distinction between non-intrusive and intrusive methods.

\subsection{Non-intrusive Methods}

First, to limit the number of calls of the deterministic model which grows exponentially with the number of random parameters, the number of quadrature points $q_{i}$ (see 3.1) can not be the same along each random direction $p_{i}(\theta)$. In fact, if a parameter $p_{i}$ has almost no influence on the variability of the quantity of interest $\mathrm{G}$ then $\mathrm{G}$ needs to be evaluated only on one quadrature point $p_{i}^{1}$ along the dimension $i$, which limits the number of evaluations $\mathrm{Q}\left(\mathrm{Q}=q_{1} \times \ldots \times q_{i-1} \times 1 \times q_{i+1} \times \ldots \times q_{K}\right)$. The number of quadrature points is optimized automatically based an error indicator which can be for example the value of the variance of the quantity of interest. Adaptive methods coupled with sparse grids and nested quadrature scheme have shown their efficiency on practical application [2]. However, with a high parameter number, the expansion based on truncated PCE becomes too large (see (14)). To limit the number of terms, a sparse basis should be constructed which can be determined from the adaptive scheme or directly from a random sampling of the quantity of interest. In [25,27], the most significant terms of the PCE are extracted using iterative algorithm aiming at reducing not only the error of approximation but also the number of terms of the expansion. These methods are efficient if a small fraction of coefficients $g_{i}$ in the exact expression (10) of the quantity of interest are dominant.

Another alternative to reduce the number of terms of the expansion is to decompose the quantity of interest under a sum of separable functions $G[\mathbf{p}(\theta)]=$

$\sum_{j=1}^{T} u_{1}^{j}\left[p_{1}(\theta)\right] \ldots u_{K}^{j}\left[p_{K}(\theta)\right]$ with $T$ the tensor rank. The functions $u_{i}^{j}\left[p_{j}(\theta)\right]$ are the unknowns of the problem and are sought in a one dimensional space for example the space generated by the polynomials $\psi_{i}^{l}\left[p_{i}(\theta)\right]$ (see (11)). The calculation of the optimal low rank approximation (the value of $T$ as smaller as possible) is a difficult task. Methods have been recently proposed in the literature to tackle this issue [37] for stochastic problems.

Finally, an adaptive interpolation technique is proposed in [38] to determine a sparse polynomial approximation using an iterative procedure. The evaluation points $\mathbf{p}_{l}$ are determined iteratively by comparing the error between the approximation and the full model. These evaluation points must satisfy an admissible condition in order to obtain interpolant polynomials. For a class of parametric elliptic problems, a fast convergence of the method has been proved. 


\subsection{Intrusive Methods}

We have seen that the application of the Galerkin Method requires the solution of a huge equation system (9) of size NxP. Under separability condition on the behaviour law, this system of equations can be written in the form of (21) which alleviates the storage space requirement. Dedicated solvers can be applied $[1,14]$ but it does not decrease the size of the equation system. Model Order Reduction Methods like Proper Orthogonal Decomposition (POD), Reduced Basis Method enables to reduce the stochastic problem (9) to solve to an order $R \leq \leq N$ ( $\mathrm{N}$ is the number of DoF's of the spatial mesh) [49]. The unknown vector $\mathbf{A}[\mathbf{p}(\theta)]$ is approximated by $\sum_{i=1}^{R} a_{i}^{r}[\mathbf{p}(\theta)] \mathbf{A}_{i}^{r}$ with $\mathbf{A}_{i}^{r}$ solutions of (9) for a given set of parameters $\left(\mathbf{p}^{1}, \ldots, \mathbf{p}^{R}\right)^{1}$. Replacing $\mathbf{A}[\mathbf{p}(\theta)]$ in (9) leads to an overdetermined system of $\mathrm{N}$ equations with $\mathrm{R}$ unknowns. Then, by applying the Galerkin method for example, a reduced equation system of $\mathrm{R}$ equations with $\mathrm{R}$ unknowns is obtained under the form $\mathbf{S}_{r}[\mathbf{p}(\theta)] \mathbf{A}^{r}[\mathbf{p}(\theta)]=\mathbf{F}[\mathbf{p}(\theta)]$. The $\mathrm{R}$ functions $a_{i}^{r}[\mathbf{p}(\theta)]$ becomes the unknowns which are then approximated by the expression (10) that is to say $a_{i}^{r}[\mathbf{p}(\theta)]=\sum_{j=0}^{\mathrm{P}} a_{i j}^{r} \Psi_{i}[\mathbf{p}(\theta)]$. The terms $a_{i j}^{r}$ can be determined by applying the methods presented in (3.2) or (3.1). This approach has been applied to solve a dosimetry problem where the reduced basis method and a non-intrusive collocation have been combined [32]. The efficiency of the model order reduction method relies on the choice of the reduced basis spanned by the $\mathbf{A}_{i}^{r}$. Error indicators, available in the literature, can help for the determination of the reduced basis.

Another approach has been proposed in [40] and applied recently in electromagnetism in [41] called the Proper Generalized Decomposition (PGD) [41]. The idea is to search a solution under the form:

$$
\mathbf{A}[\mathbf{x}, \mathbf{p}(\theta)]=\sum_{i=1}^{T} a_{i}^{P G D}[\mathbf{p}(\boldsymbol{\theta})] \mathbf{A}_{i}^{P G D}(\mathbf{x})
$$

with $\mathbf{A}_{i}^{P G D}(\mathbf{x})$ in $W(D)$ and $a_{i}^{P G D}[\mathbf{p}(\theta)]$ in $\mathrm{P}_{P}^{K}$ (see (3.2)). The couple of functions $\left(a_{i}^{P G D}[\mathbf{p}(\theta)], \mathbf{A}_{i}^{P G D}(\mathbf{x})\right)$ is determined iteratively from the previous couples $\left(a_{j}^{P G D}[\mathbf{p}(\theta)], \mathbf{A}_{j}^{P G D}(\mathbf{x})\right) 1 \leq j \leq i-1$. The process is stopped when the contribution of the couple $\left(a_{i}^{P G D}[\mathbf{p}(\boldsymbol{\theta})], \mathbf{A}_{i}^{P G D}(\mathbf{x})\right)$ is "sufficiently" small. The term $a_{i}^{P G D}[\mathbf{p}(\boldsymbol{\theta})]$ satisfies a system of $\mathrm{P}$ equations which depends on the terms $\mathbf{A}_{i}^{P G D}(\mathbf{x})$ and the term $\mathbf{A}_{i}^{P G D}(\mathbf{x})$ a system of $\mathrm{N}$ equations which depends on the functions $a_{i}^{P G D}[\mathbf{p}(\boldsymbol{\theta})]$. The determination of $\left(a_{i}^{P G D}[\mathbf{p}(\theta)], \mathbf{A}_{i}^{P G D}(\mathbf{x})\right)$ requires the solution of two coupled equation systems of size $\mathrm{N}$ and $\mathrm{P}$ which are usually solved iteratively using a fixed point method. If $\mathrm{T}$ couples are required we can see that we have only $\mathrm{T} \times(\mathrm{N}+\mathrm{P})$ unknowns instead of NxP in the Galerkin approach (see (3.2)). If the number T of couples to approximate correctly the solution is small, this method is very interesting in terms of memory storage and computation time. Moreover, under "separabilty" conditions

\footnotetext{
${ }^{1}$ The vectors $\mathbf{A}_{i}^{r}$ must be linearly independent to enforce the uniqueness of the solution of the reduced problem. If it is not the case, a Singular Value Decomposition (SVD) or a Gram-Schmidt process can be applied to obtain linearly independent vectors
} 
on the behaviour law (see ), the term $a_{i}^{P G D}[\mathbf{p}(\theta)]$ can be sought under the following separable form:

$$
a_{i}[\mathbf{p}(\boldsymbol{\theta})]=\prod_{j=1}^{K} a_{i j}^{P G D}\left[p_{j}(\boldsymbol{\theta})\right]
$$

Then $a_{i}[\mathbf{p}(\theta)]$ is obtained by solving $\mathrm{K}$ one dimensional problems which avoid the curse of dimensionality when the number $\mathrm{K}$ of parameters is too large. The PGD remains intrusive in the sense that, to be implemented, numerous additional developments in a deterministic software are required. However, recently, a method has been proposed to compute an approximation of the solution based on simple evaluations of the residual of the deterministic problem [43].

\section{Conclusion}

In this paper, we have presented approximation methods to solve stochastic problems based on partial differential equations. Examples of application in computational electromagnetism have been presented showing that the stochastic approach based on approximation methods provide very useful tools for the study and the design of electromagnetic devices. It has been shown that when the number of random parameters is high, the approximation can leads to an unsolvable problem (curse of dimensionality). To face this issue, recent methods proposed in the literature have been listed.

\section{Acknowledgement}

This work has been supported by the pole MEDEE funded by the Nord Pas de Calais Region and the European Union and also by the Arts et Métiers Fundation.

\section{References}

1. Beddek, K.; Clénet, S.; Moreau, O.; Le Menach, Y., Solution of Large Stochastic Finite Element Problems Application to ECT-NDT, IEEE Transactions on Magnetics, 49:5, pp. 16051608,2013

2. Beddek, K.; Clénet, S.; Moreau, O.; Costan, V.; Le Menach, Y.; Benabou, A.; Adaptive method for non-intrusive spectral projection application on a stochastic eddy current NDT problem, IEEE Transactions on Magnetics, 48:2, pp. 759-762, 2012

3. Gaignaire, R.; Clnet S.; Moreau, O; Guyomarch, F.; Sudret, B, Speeding up in SSFEM Computation using Kronecker Tensor Products, IEEE Transactions on Magnetics, 45:3, pp. 14321435,2009 
4. Matthies, H.G.; Keese, A., Galerkin method for linear and non-linear elliptic stochastic partial differential equations, Computional Methods Applied Mechanical Engineering, 194, pp. 12951331,2005

5. Xiu, D.; Tartakovsky, D.M., Numerical methods for differential equations in random domains, SIAM Journal on Scientific Computing, 3, pp. 1167-1185, 2006

6. Mac, H.; Clénet, S.; Mipo, J.C., Comparison of two approaches to compute magnetic field in problems with random domains, IET Science, Measurement and Technology, 6:5, pp. 714721,2012

7. Mac, H.; Clénet, S.; Mipo, J.C., Transformation method for static field problem with Random Domains, IEEE Transactions on Magnetics, 47:5, pp. 1446-1449, 2011

8. Mac, H.; Clénet, S.; Mipo, J.C.; Moreau, O., Solution of Static Field Problems with Random Domains, IEEE Transactions on Magnetics, 46:8, pp. 3385-3388, 2010

9. Nouy, A.; Clément, A., eXtended Stochastic Finite Element Method for the numerical simulation of heterogeneous materials with random material interfaces, International Journal For Numumerical Methods In Engineering, 83:10, pp. 1312-1344, 2010

10. Nouy, A.; Clément, A.; Schoefs, F.; et al., An extended stochastic finite element method for solving stochastic partial differential equations on random domains, Computational Methods in Applied Mechanics and Engineering, 197:51-52, pp 4663-4682, 2008

11. Hammersley, J. M.; Handscomb, D. C. , Monte Carlo Methods, Chapman and Hall, London and New York, 1964.

12. Metropolis, N; Rosenbluth, A.W., Rosenbluth, M.N.; Teller, H.A.; Teller, E, Equation of state calculations by fast computing machines, Journal of Chemical Physics, 21:6, pp. 1087-1092, 1953

13. Le Maitre O. P.; Knio O.M.; Najm H.N.; Ghanem R.G., Uncertainty propagation using Wiener-Haar expansions, Journal of computational Physics, 197, pp. 28-57, 2004

14. Le Maitre, O.; Knio, O.M., Spectral Methods for Uncertainty Quantification with Applications to Computational Fluid Dynamics, Springer Series Scientific Computation

15. Babuska, I; Tempone, R; Zouraris, E, Galerkin Finite Element Approximation of Stochastic Elliptic Partial Differential Equations, SIAM Journal of Numerical Analysis, 42:2, pp. 800825, 2004

16. Weiner, N., The homogeneous chaos, American Journal of Mathemetics, 60:4, pp. 897-936, 1938

17. Xiu, D.; Karniadakis, G, The Wiener Askey polynomial chaos for stochastic differential equations, SIAM Journal of Scientific Computing, 24:2, pp. 619-644.

18. Gaignaire, R.; Scorretti, R.; Sabariego, R.V.; Geuzaine, C, Stochastic Uncertainty Quantification of Eddy Currents in the Human Body by Polynomial Chaos Decomposition,IEEE Transaction on Magnetics, 48:2, pp. 451454, 2012

19. Gaignaire, R.; Crevecoeur, G.; Dupreé, L.; Sabariego, R.V.; Dular, P.; Geuzaine, C., Stochastic Uncertainty Quantification of the Conductivity in EEG Source Analysis by Using Polynomial Chaos Decomposition IEEE Transaction on Magnetics, 46:8, pp. 34573460, 2010

20. Liu M.; Gao, Z. ; Hesthaven J.S., Adaptive sparse grid algorithms with applications to electromagnetic scattering under uncertainty; Applied Numerical Mathematics, 61:1, pp. 24-37, 2011

21. Deryckere, J.; Masschaele, B.; De Gersem, H.; Steyaert, D., Stochastic Response Surface Method for Dimensioning Accelerator Cavities, OIPE 2012, Gent (Belgium), 2012

22. Voyer, D.; Musy, F.;Nicolas, L.; Perrussel, R., Probabilistic methods applied to 2D electromagnetic numerical dosimetry, The International Journal for Computation and Mathematics in Electrical and Electronic Engineering, 27:3, pp. 651-667, 2008

23. Smolyak, S., Quadrature and Interpolation Formulas for Tensor Products of Certain Classes of Functions,, Doklady Akademii Nauk SSSR, 4, pp. 240-243, 1963

24. Berveiller, M. ; Sudret, B. ; Lemaire, M., Stochastic finite elements: a non-intrusive approach by regression. European Journal of Computional Mechanics, 15:(1-3), pp. 8192, 2006

25. Doostan, A.; Owhadi, H., A non-adapted sparse approximation of PDEs with stochastic inputs, Journal of Computational Physics, 230, pp. 30153034, 2011 
26. Clénet, S.; Ida, N.; Gaignaire, R.; Moreau, O.; Solution of dual stochastic static formulations using double orthogonal polynomials of Static Field, IEEE Transactions on Magnetics, 46:8, pp. 3543-3546, 2010

27. Blatman, G.; Sudret, B.; Adaptive sparse polynomial chaos expansion based on least angle regression, Journal of Computational Physics, 230:6, pp. 2345-2367, 2011

28. Rosseel, E.; De Gersem, H.; Vandewalle, H., Spectral Stochastic Simulation of a Ferromagnetic Cylinder Rotating at High Speed IEEE Transactions on Magnetics, 47:5, pp. 1182-1185, 2011.

29. Mac, H.;Clénet, S.; Zheng, S.; Coorevits, T.;Mipo, J.C., On the geometric uncertainties of an electrical machine : stochastic modeling and impact on the performances, COMPUMAG 13, Budapest (Hungary), 2013

30. Offermann, P.; Mac, H.;Nguyen, T.T.;Clénet, S.;De Gersem, H.; Hameyer, K., Uncertainty quantification and sensitivity analysis in electrical machines with stochastically varying machine parameters, CEFC 14, Grenoble (France), 2014.

31. Ghanem, R.; Spanos, P. D., Stochastic Finite Elements: A spectral approach, Dover, New York, 2003.

32. Drissaoui, A.; Lanteri, S.;Lévłque, P.;Musy, P.;Nicolas, L.;Perrussel, R.;Voyer, D., A Stochastic Collocation Method Combined With a Reduced Basis Method to Compute Uncertainties in Numerical Dosimetry, IEEE Transactions on Magnetics , 48:2, pp 563-566, 2012

33. Fratila, M.; Ramarotafika, R.; Benabou, A.; Clénet, S.; Tounzi, A., Stochastic post-processing calculation of iron losses application to a PMSM, COMPEL, 32:4, pp. 1383-1392, 2013

34. Moreau, O.; Beddek, K.; Clénet, S.; Le Menach, Y., Stochastic Non Destructive Testing simulation: sensitivity analysis applied to material properties in clogging of nuclear power plant steam generator, IEEE Transactions on Magnetics, 49:5, pp. 1873-1876, 2013

35. Mac, $\mathrm{H}$. et al, Influence of uncertainties on the $\mathrm{B}(\mathrm{H})$ curves on the flux linkage of a turboalternator, IJNM, 27, pp. 385-399, 2014

36. Kim, Y.; Hong, J.; Hur, J., Torque Characteristic analysis Considering the Manufacturing Tolerance for Electric Machine by Stochastic Response Surface Method, IEEE Transactions on Industry Applications, 39:3, pp. 713-719, 2003

37. Rai, P.; Chevreuil, M.; Nouy, A.;Lebrun, R., A regression based method using sparse low rank approximations for uncertainty propagation, 7th International Conference on Sensitivity Analysis of Model Output-SAMO 2013

38. Chkifa, A.; Cohen, A.; Schwab, C., High-dimensional adaptive sparse polynomial interpolation and application to parametric PDEs, JfoCM 14, pp. 601-633, 2014

39. Ramarotafika R.; Benabou A.; Clénet, S.; Mipo J.C., Experimental characterization of the iron losses variability in stators of electrical machines, IEEE Transaction on Magnetics , 48:4, pp. 629-1632, 2012

40. Nouy, A., A generalized spectral decomposition technique to solve a class of linear stochastic partial differential equations, Computational Methods in Applied Mechanical Engineering, 196:3740, pp. 45214537, 2007

41. Codecasa, L.; Di Rienzo, L.; Generalised spectral decomposition approach to a stochastic finite integration technique electrokinetic formulation, CEM 2014, London, 2014

42. Chinesta, F.; Ladeveze, P.; Cueto, E.; A Short Review on Model Order Reduction Based on Proper Generalized Decomposition. Archives of Computational Methods in Engineering, 18:4, pp. 395404, 2011

43. Giraldi, L.; Liu, D.; Matthies, H.G.; Nouy, A. To be or not to be intrusive? The solution of parametric and stochastic equations Proper Generalized Decomposition, arXiv:1405.0875v1 [math.NA], 2014

44. Harbrecht, H; Schneider, R; Schwab C., Sparse second moment analysis for elliptic problems in stochastic domains, Numerische Mathematik, 109, pp. 385414, 2008

45. Romer, U.; Schops, S.; Weiland, T., Approximation of moments for the nonlinear manetoquasistatics problem with material uncertainties. IEEE Transactions on Magnetics, 50:2, pp. 417420, 2014.

46. Rosseel E.; Vandewalle S., Iterative solvers for the stochastic finite element method. SIAM J. Sci. Comput., 32:1, pp. 372397, 2010 
47. Schmidt C.; Flisgen T.; Heller T.; Van Rienen U., Comparison of techniques for uncertainty quantification of superconducting radio frequency cavities, International Conference on Electromagnetics in Advanced Applications 2014 (ICEAA 2014), pp. 117-120, 2014

48. Bartel A.; De Gersem H.; Hlsmann T.; Romer U.; Schops S.; Weiland T.; Quantification of uncertainty in the field quality of magnets originating from material measurements. IEEE Transactions on Magnetics, 49:5, pp. 2367-2370, 2013

49. Haasdonk, B.; Urban, K.; Wieland, B.; Reduced basis methods for parameterized partial differential equations with stochastic influences using the Karhunen-Loeve expansion, Journal of Uncertainty Quantification, 1:1, pp. 79-105, 2013

50. Sobol, I.M., Sensitivity estimates for non linear mathematical models and their Monte Carlo estimates, Mathematics and Computers in Simulation, 55, pp. 271280, 2001

51. Crestaux, T.; Le Maitre, O.; Martinez, J.M., Polynomial chaos expansion for sensitivity analysis, reliability engineering and system safety, 94:7, pp. 1161-1172, 2009

52. Sudret, B.; Global sensitivity analysis using polynomial chaos expansions. Reliability Engineering and System Safety, 93, pp. 964979, 2008 